\title{
DESEMPENHO SOCIAL E FINANCEIRO DE PEQUENAS E MÉDIAS EMPRESAS: MODELO CONCEITUAL DE CAUSA E EFEITO ${ }^{1}$
}

\section{RESUMO}

Desempenho social empresarial pode ser entendido como uma medida de mensuração de eficiência das interações mantidas pelas empresas com seus stakeholders principais. Sua avaliação tem resultado em avanços nas pesquisas e em implicações gerenciais. Um de seus temas centrais, o estudo da relação entre desempenho social e financeiro, tem como foco tradicional empresas de capital aberto, fato que revela na literatura uma lacuna em termos de objeto. Investigar pequenas e médias empresas (PMEs) justifica-se pela relevância econômica e social delas e pelas novas evidências que podem vir desses estudos. Após revisão da literatura, este texto apresenta um modelo conceitual composto por proposições de pesquisa para serem testados por futuros estudos empíricos que desejem responder a seguinte questão: em pequenas e médias empresas existem relações de causa e efeito entre desempenho social e financeiro? $\mathrm{O}$ teste das proposições sugeridas neste texto pode revelar, dentre outros resultados, as categorias do desempenho social de PMEs mais afetadas por um desempenho financeiro superior, conforme estabelecido pelas premissas da teoria slack-resources; se o impacto dessas categorias sobre o desempenho financeiro é qualificado pela forma de gestão, confirmando pressupostos da teoria good management, ou se não há diferenças significativas entre o desempenho social de PMEs com desempenho financeiro superior e o de PMEs com desempenho financeiro inferior, revelando a existência de fatores não financeiros que também influenciam o desempenho social.

Palavras-Chaves: Desempenho social e financeiro, estratégia empresarial, pequenas e médias empresas.

\section{ABSTRACT}

Corporate social performance can be understood as a way to measure the efficiency of interactions between companies and their main stakeholders. This evaluation has led to some steps forward in research and management implications. One of its main issues, which is the study of the relationship between social and financial performance, focuses on traditional joint-stock companies. This fact reveals a gap concerning the object of study in the literature of the area. The importance of investigating small and medium companies (SMCs) lies in their social and economic relevance and also in new evidences these studies may provide. After the theoretical discussion, this study presents a conceptual model composed of research propositions to be tested by future empirical studies that wish to answer the following question: in small and medium companies there are relations of cause and effect between social and financial performance? The test of the proposals suggested can reveal, among other results, the categories of social performance of SMCs most affected by a higher financial performance, as established by the premises of theoretical slack-resources; if the impact of these categories on the financial performance is qualified by way of management, confirming assumptions of the theory good management, or if there are no significant differences between the social performance of SMEs with higher financial performance and SMEs with low financial performance, revealing the existence of non-financial factors also influence social performance.

Key Words: Social and financial performance, business strategy, small and medium companies.

\footnotetext{
${ }^{1}$ Este trabalho tem apoio do Conselho Nacional de Desenvolvimento Científico e Tecnológico (CNPQ).

${ }^{2}$ Instituto Federal de Brasília -IFB, Brasil

E-mail: elenilsoncruz@hotmail.com

${ }^{3}$ Universidade de Brasília-Unb, Brasil

E-mail: rbarreirosporto@gmail.com>
} 


\section{INTRODUÇÃO}

Apesar de ser um tema permeado por perspectivas teóricas divergentes, responsabilidade social empresarial (RSE) pode ser conceituada como o conjunto das expectativas econômicas, legais e éticas que a sociedade tem das empresas em determinado momento (Carroll, 1979, 1991; Schwartz \& Carroll, 2003). Esse conceito indica que as responsabilidades das empresas não estão restritas apenas aos seus acionistas, mas expandem-se para toda a sociedade, de forma geral. A partir do consenso quanto ao significado e relevância da RSE, surgiram os estudos em torno do desempenho social empresarial (Rowley \& Berman, 2000). O foco desses estudos é testar e operacionalizar a noção de responsabilidade social das empresas e descrever os grupos constituintes que elas devem honrar no desenvolvimento de suas atividades (Rowley \& Berman, 2000).

Desempenho social empresarial pode ser entendido como uma medida de mensuração de eficiência das interações mantidas pelas empresas com seus stakeholders principais (Waddock \& Graves, 1997). Sua avaliação tem-se demonstrado relevante tanto para a academia quanto para as empresas. Aos acadêmicos tal avaliação permite identificar as relações entre as diferentes medidas organizacionais e o próprio desempenho social, e aos gestores ela possibilita empregar as informações do desempenho social em seus modelos de tomada de decisão (Ruf, Muralidhar, \& Paul, 1998). Inseridos no primeiro contexto, estão os estudos das relações entre desempenho social e desempenho financeiro empresarial e suas possíveis correlações de causa e efeito. Tais estudos, tradicionalmente realizados com empresas de capital aberto, não têm investigado essas relações em pequenas e médias empresas (PMEs), apesar da relevância econômica e social dessas empresas, caracterizada por representatividade numérica, capacidade de geração de emprego e capilaridade geográfica (Raynard \& Forstater, 2002; Koteski, 2004).

Ao se propor investigar as relações entre desempenho social e financeiro em PMEs, recomendase às futuras pesquisas avançar sobre lacunas teóricas, em especial as derivadas das teorias slack-resources e good management (Waddock \& Graves, 1997). Para tanto, trabalhos empíricos devem considerar as significativas diferenças existentes entre PMEs e empresas de grande porte (Spence, 1999, Jenkins, 2004, 2006) e alterar a forma de classificação das ações de RSE, visando minimizar os efeitos das limitações do modelo conceitual de RSE, de Schwartz \& Carroll (2003), e adaptá-lo mais adequadamente ao contexto de PMEs. Assim sendo, a mensuração do desempenho social de PMEs pode ser empreendida em termos de ações obrigatórias ou espontâneas, conforme será discutido na fundamentação teórica. Procedendo assim, pesquisadores podem testar com melhor nível de análise os pressupostos da teoria slack-resources, investigando se PMEs com desempenho financeiro superior apresentam desempenho social espontâneo maior do que PMEs com desempenho financeiro inferior.

E, nesse caso, investigações podem estender-se para identificar as categorias do desempenho social afetadas mais significativamente por empresas que apresentam desempenho financeiro superior, conforme sugerido por Melo (2012). Ao se identificar tais categorias, pode-se aplicar os pressupostos da teoria good management para investigar se o retorno econômico dessas categorias vincula-se à forma de gestão, uma vez que defensores da teoria good management (Mcwilliams \& Siegel, 2000; Brammer \& Pavelim, 2006; Porter \& Krammer, 2006) afirmam que ações de RSE revertem-se em ganhos econômicos se forem geridas de forma estratégica.

Nesse aspecto, após revisão da literatura, esse texto tem por objetivo apresentar um modelo teórico composto por proposições de pesquisa para serem testados por futuros estudos empíricos que desejem responder à seguinte questão: em pequenas e médias empresas existem relações de causa e efeito entre desempenho social e financeiro? Espera-se que os testes das proposições sugeridas neste texto possam revelar, dentre outros resultados, as categorias do desempenho social de PMEs mais afetadas por um desempenho financeiro superior, conforme estabelecido pelas premissas da teoria slack-resources; se o impacto dessas categorias sobre o desempenho financeiro é qualificado pela forma de gestão, confirmando pressupostos da teoria good management, ou se não há diferenças significativas entre $o$ desempenho social de PMEs com desempenho financeiro superior e o de PMEs com desempenho financeiro inferior, revelando a existência fatores não financeiros que também influenciam o desempenho social.

Este texto está estruturado em cinco partes, incluindo esta introdução. Na segunda parte, são discutidos os principais aspectos teóricos norteadores do estudo. Na terceira são apresentadas as proposições de pesquisa e o modelo conceitual. Na quarta são apresentadas as discussões e notas finais, destacando as implicações teóricas e gerenciais dos possíveis resultados. 


\section{FUNDAMENTAÇÃO TEÓRICA}

\subsection{Abordagem estratégica e modelo conceitual de Responsabilidade social empresarial}

Este trabalho é desenvolvido sob a perspectiva da abordagem estratégica da RSE, corrente teórica que tem procurado operacionalizar a noção de responsabilidade social das empresas por meio do desempenho social (Rowley \& Berman, 2000), produzir instrumentos de gestão objetivando melhorar o desempenho social, prescrever como as empresas podem auferir vantagens competitivas a partir de desempenho social superior e investigar possíveis

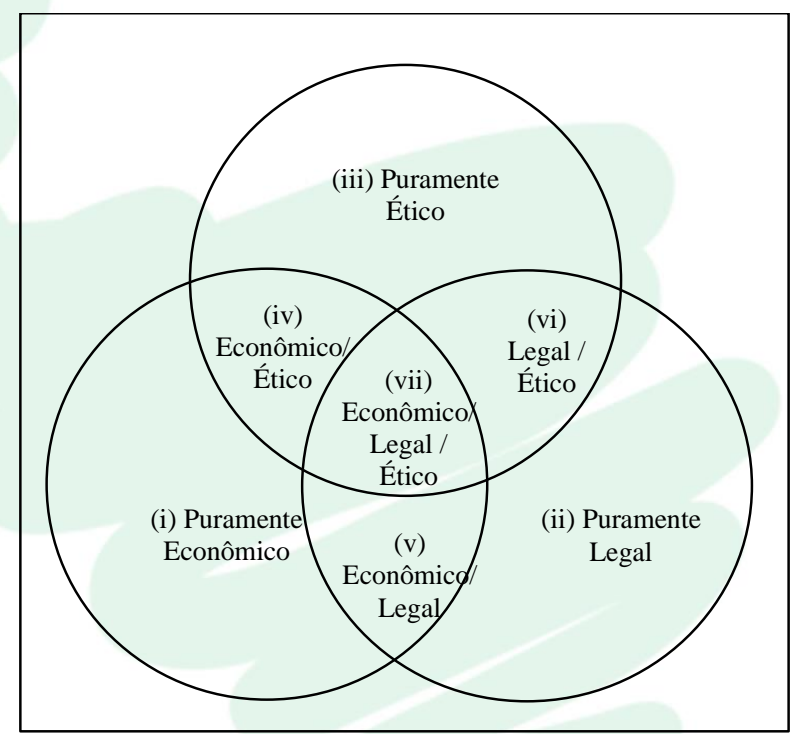

Figura 1: modelo dos três domínios da RSE

Fonte: Schwartz e Carroll (2003, p. 519)

Como tais domínios se sobrepõem em um ou mais aspectos, a RSE é uma configuração de atividades inseridas em sete subcategorias do modelo. No entanto, a sétima categoria (econômica/legal/ética), localizada no centro do modelo, apresenta simultaneamente entrelaçadas as responsabilidades econômicas, legais e éticas (Schwartz \& Carroll, 2003). É a categoria ideal, na qual as empresas devem atuar sempre que possível. Além de representar por completo a perspectiva da RSE, essa categoria está alinhada com o modelo de gestão triple bottom line, cujas premissas contemplam as dimensões econômica, social e ambiental do desenvolvimento sustentável no âmbito das empresas (Barbieri \& Cajazeira, 2009).

Uma das limitações desse modelo é a dificuldade de aplicação de práticas puramente econômicas, legais e éticas, uma vez que em algum aspecto ou extensão elas se sobrepõem, como pode ser visto nas categorias iv (econômico/ético), v (econômico/legal) e vi (legal/ético). Ações puramente relações de causa e efeito entre desempenho social e financeiro (Faria \& Sauerbronn, 2008).

Os estudos em torno da RSE foram intensificados a partir da década de 1950, tendo por objetivo melhor delinear seus contornos e compreender com mais acuidade as motivações do engajamento das empresas (Carroll, 1979). Revisando os intensos debates sobre RSE, Archie B. Carroll, após alguns aperfeiçoamentos, encerrou um modelo conceitual de RSE, integrando os diversos posicionamentos teóricos sobre o tema. Nesse modelo, RSE abrange o conjunto das expectativas econômicas, legais e éticas que a sociedade tem das empresas em determinado momento. O modelo é composto por três domínios: econômico, legal e ético, conforme demonstra a figura 1:

econômicas podem estar de acordo com a lei e serem suportadas por algum padrão ético; ações puramente legais acabam tendo implicações econômicas, como aquelas que geram perdas para as empresas; ações puramente éticas podem ter consequências econômicas (positivas ou negativas) e serem passivamente legais (Schwartz \& Carroll, 2003). Isso indica que em algum momento, a maioria das práticas empresariais cairá dentro do domínio econômico.

Tal limitação pode ser mais acentuada no contexto das PMEs devido às suas significativas diferenças em relação às grandes empresas. PMEs são heterogêneas em tamanho, capacidade de recursos, relações pessoais, controle e cultura (Spence, 1999; Jenkins, 2004). Tais diferenças se refletem num estilo de gestão caracterizado pela informalidade, confiança, intuição e monitoramento pessoal do proprietário. Por isso é tão comum em PMEs uma confusão entre aspectos organizacionais e pessoais (Jenkins, 2004). 
Nesse aspecto, este estudo sugere às futuras pesquisas empíricas adaptarem o modelo conceitual de Schwartz e Carroll (2003) para classificar as ações de RSE em PMEs em duas naturezas: obrigatórias e espontâneas, independentemente, de terem ou não finalidade econômica. Como ações obrigatórias devem ser consideradas aquelas previstas em leis, decretos, regulamentos e portarias emitidas por órgãos públicos reguladores, bem como as pacificadas em jurisprudências. Práticas espontâneas serão todas as demais realizadas pela empresa, em favor de seus stakeholders, cuja realização é feita pela empresa sem qualquer constrangimento de ordem legal.

\subsection{Evolução dos estudos em desempenho social empresarial e delimitação do conceito}

A evolução dos estudos sobre desempenho social empresarial é marcada por algumas contribuições conceituais importantes. Em 1978 William C. Frederick afirmou que o termo responsividade social corporativa reflete mais adequadamente a ação gerencial, orientada e instrumentalizada com técnicas administrativas, para verificar até que ponto as empresas irão responder às pressões sociais (Frederick, 1994).

Para Sethi (1979), desempenho social é sinônimo de legitimidade empresarial, que aumenta conforme a empresa consegue reduzir a lacuna entre seu desempenho e as expectativas da sociedade. Nesse ponto de vista, desempenho social também é descrito como uma medida do comportamento da empresa, que é configurado em forma de três estágios de evolução. $\mathrm{O}$ primeiro, obrigação social, diz respeito ao comportamento reativo às forças de mercado e às restrições legais. O segundo, responsabilidade social, refere-se a um tipo de comportamento prescritivo, porém alinhado com os valores, normas e expectativas da sociedade. No terceiro estágio, responsividade social, o comportamento da empresa é antecipatório, proativo e preventivo por natureza (Sethi, 1979).

Para Wartick e Cochran (1985), desempenho social empresarial é uma interação subjacente entre os princípios de responsabilidade social, processo de responsividade social e políticas criadas para tratar de temas sociais. Em 1991, Dona Wood revisa esse conceito ao inserir uma referência a ações e resultados - dois componentes que constituem o significado do termo desempenho. Para Wood (1991), tais componentes são evidenciados pela existência de políticas e programas na empresa e pelos resultados observáveis dos impactos que tais políticas e programas provocam nas relações da empresa com seus stakeholders.

Para Waddock e Graves (1997), desempenho social é uma medida de mensuração de eficiência das interações mantidas pelas empresas com seus stakeholders principais e está vinculado ao desempenho da própria gestão. Nessa visão, as despesas realizadas nos domínios dos stakeholders principais, além de melhorar o próprio desempenho social, também contribuem para a melhoria do desempenho organizacional como um todo (Waddock \& Graves, 1997).

Para fins de operacionalização, este trabalho sugere aos futuros estudos empíricos que $o$ desempenho social de PMEs seja mensurado por ações direcionadas ao atendimento das demandas dos stakeholders, obrigatórias ou espontâneas, e expressas em resultados econômicos e não econômicos.

\subsection{As dimensões do desempenho social exploradas em PMEs}

As dimensões do desempenho social variam de acordo com o contexto do objeto de estudo e objetivo dos pesquisadores. Estudos com PMEs revelam as dimensões mais frequentes. No trabalho de Princic e Floyd (2003) as dimensões são empregados, meio ambiente e comunidade. Jenkins (2006) as classifica em empregados, clientes, fornecedores e comunidade. Para Brammer, Hoejmose, \& Marchant (2012), as dimensões são empregados, processos produtivos, corporação e marketing. A revisão de literatura de Vo (2011) aponta para meio ambiente, comunidade e empregados.

Para fins de delimitação das dimensões que podem ser exploradas por futuros tralhos empíricos com PMEs, convém destacar que empregados e clientes são considerados stakeholders primários (Van Der Laan, Van Ees, \& Van Witteloostuijn, 2008). Investimentos realizados nas demandas desses stakeholders contribuem para melhorar o desempenho financeiro das empresas (Van Der Laan et al., 2008).

Adicionalmente, a comunidade, por manter relações muito próximas com PMEs (Jenkis, 2006), torna essas empresas mais sensíveis aos problemas comunitários (Vo, 2011). Isso leva PMEs a envolverem-se mais efetivamente em eventos comunitários e priorizarem a contratação de mão de obra e de fornecedores locais (Jenkins, 2006). Além disso, proprietários de PMEs acreditam que o atendimento aos interesses da comunidade impacta na boa imagem da empresa e no retorno dos investimentos (Oliveira, 1984).

Assim, este estudo considera que os stakeholders mais relevantes às PMEs são empregados, comunidade e clientes. Por conta disso, sugere-se que tais stakeholders representem as dimensões do desempenho social a serem investigadas por futuras pesquisas. Quanto aos temas e subtemas que compõem tais dimensões, trabalhos empíricos podem ter como base os temas previstos no balanço social, modelo IBASE, e nos indicadores Ethos de RSE, versão para micro e pequenas empresas. 
2.4 Desempenho Financeiro em PMEs: dificuldades enfrentadas por PMEs

O quadro recessivo instalado na economia brasileira nos últimos anos vem afetando negativamente o ritmo dos negócios e a geração de caixa das empresas. Como consequência, empresas enfrentam problemas financeiros e dificuldades para honrar seus compromissos. Nos últimos anos, mais de 90\% das PMEs enfrentaram problemas financeiras (Campos, 2014), corroborando a premissa de que PMEs são altamente vulneráveis frente às mudanças ambientais (Campos, Nishimura, Ramos, Cherez, \& Scalfi, 2008). Nessa situação, PMEs esbarram em falta de crédito e enfrentam elevadas taxas de juros (Brigham \& Houston, 1999).

Além disso, é comum PMEs operarem com prejuízos persistentes, apresentarem baixas taxas de lucro e incorrem em atraso sistemático de pagamentos de impostos, encargos sociais e outras obrigações (Lucato \& Vieira Júnior, 2006). Outras características de PMEs em dificuldades financeiras é a falta de capacidade para investir em novos produtos e processos e contratar talentos gerenciais (Buckley, 1989). Ainda segundo Bucley (1989), empresas em dificuldades financeiras acabam por tomar decisões de redução de custos extremamente danosas às operações, tais como cortes de salários de pessoas-chave e redução de gastos em processos de adaptação de produtos e na aquisição de informações mercadológicas. Essas decisões acabam por afastar fatores essenciais para a redução da vulnerabilidade ambiental que atinge tais empresas (Buckley, 1989).

\subsection{Mensuração do desempenho financeiro: dimensões e indicadores}

Segundo Combs, Crook e Shook (2005), o desempenho financeiro é composto por três dimensões: retorno contábil, crescimento e valor de mercado. Para esses autores, as medidas principais que refletem o retorno contábil são: retorno sobre os ativos (ROA) e retorno sobre o investimento (ROI). Dentre as medidas de crescimento, as mais utilizadas são: crescimento das vendas, crescimento do lucro e participação de mercado. Dos indicadores de valor de mercado, os mais comuns são: retorno sobre ação e Q de Tobin. Combs et al., (2005) sugerem evitar o uso de medidas que capturem dados simultaneamente de desempenhos diferentes, como é o caso do ROE, que combina retorno contábil - lucro (uma medida de desempenho financeiro) - com estrutura de capital - cadeia de valor (um resultado do desempenho operacional).

Em estudos sobre desempenho social e financeiro, medidas de retorno contábil estão presentes num maior número de trabalhos, enquanto medidas de valor de mercado aparecem menos frequentemente. Entretanto, os resultados obtidos com medidas de retorno contábil são mais estabilizados e menos contraditórios do que aqueles obtidos com medidas de valor de mercado (Griffin \& Mahon, 1997; Crisóstomo, Freire, \& Vasconcellos, 2011). Considerando que a utilização de medidas de base de mercado para mensuração do desempenho financeiro de PMEs é dificultada pela baixíssima participação dessas empresas no mercado de capital, este trabalho sugere às futuras pesquisas utilizar medidas de retorno contábil e de crescimento.

\subsection{Estratégia empresarial: tipos e aplicação em estudos de RSE em PMEs}

Os estudos sobre estratégia, publicados desde o início dos anos 1930, apresentam um expectro de conteúdo bastante diversificado. Ramos-Rodríguez e Ruíz-Navarro (2004) destacam, além dos autores mais influentes, os temas mais relevantes dessa área. Dentre os inúmeros temas estão: estrutura corporativa, processos de elaboração de estratégias, recursos e capacidades organizacionais, desempenho, formação de alianças, cooperação, custo de transação, custo de agência, vantagem competitiva e modelos concorrenciais. Ramos-Rodríguez e Ruíz-Navarro (2004) demonstram que compreender o campo da estratégia ou gestão estratégica significa compreender os vários temas tratados pela disciplina nos mais diferentes contextos de mercados.

Em termos conceituais, Mintzberg, Lampel, Quinn, \& Ghoshal (2006) decrevem a estratégia em forma de 5 Ps: plano, pretexto, padrão, posição e perspectiva. Como plano, estratégia revela algum tipo de ação conscientemente pretendido, uma diretriz ou um conjunto de diretrizes para lidar com uma determinada situação. Como pretexto, a etratégia revela-se como uma manobra específica para enganar o concorrente. A estratégia como padrão é aquela revelada por uma corrente de ações, um comportamento resultante consistente ao longo do tempo, pretendido ou não (Mintzberg et al., 2006).

Estratégia como posição equipara-se a um olhar para fora à procura de localizar a empresa em seu ambiente; é também uma força mediadora ou combinação entre organização (contexto interno) e ambiente (contexto externo). Como perspectiva, estratégia refere-se à forma de os estrategistas ver o mundo, a qual coloca a empresa numa posição escolhida. A estratégia como perspectiva representa para a empresa aquilo que a personalidade é para o indivíduo (Mintzberg et al., 2006).

Inúmeros estudos têm debatido o alinhamento da RSE com a estratégia empresarial, pois, muitas empresas têm empreendido ações buscando melhorar as consequências sociais e ambientais de suas atividades (Porter \& Kramer, 2006). Nesse aspecto, estudos brasileiros vinculam a RSE à estratégia organizacional como um processo formal e deliberado 
e procuram identificar, entender, avaliar e explicar como o alinhamento estratégico da RSE gera vantagens competitivas e cria valor para as organizações (Serra, Albernaz, \& Ferreira, 2007; Volpon \& Macedo-Soares, 2007; Esteves, Silva, \& Aligleri, 2007).

No entanto, pelas definições de estratégia, PMEs podem percebê-la além de um processo formal e deliberado, mas também como um processo emergente e realizado (Mintzberg, et al., 2006). Isso leva estratégias a fazerem parte de uma organização sem que elas sejam pretendidas conscientemente ou formuladas (Mintzberg, et al., 2006). Nesse caso, cabe lembrar que para Mintzberg (1973), a presença de estratégias em pequenas empresas não está vinculada à existência do planejamento como um processo racional.

Dada a diversidade de conceitos e entendimentos de estratégia, esse trabalho propõe às futuras pesquisas oportunizar aos empresários/gestores a indicação das categorias/temas de RSE que eles entendem serem estratégicos para o negócio.

\section{PROPOSIÇÕES DE PESQUISA E MODELO CONCEITUAL}

\subsection{Implicações da Teoria Slack-Resources}

A noção de que a rentabilidade gera uma folga de recursos que pode ser dedicada à responsabilidade social está implícita no argumento de que fazer "bem" (desempenho financeiro) permite fazer o "bom" (desempenho social) (Waddock \& Graves, 1997). Nesse aspecto, slack-resources refere-se a uma maior disponibilidade de recursos organizacionais (financeiros, humanos, materiais etc), consequência de desempenho financeiro superior no passado (Waddock \& Graves, 1997).

Os defensores da teoria slack-resources argumentam que os gestores empresariais têm a discricionariedade de utilizar parte desses recursos em ações de responsabilidade social que não trazem retorno imediato (Waddock; Graves, 1997), como aquelas classificadas no domínio ético do modelo conceitual de Schwartz e Carroll (2003), que para fins deste trabalho, são classificadas como espontâneas. Nesse caso, desempenho financeiro torna-se preditor de desempenho social (Waddock \& Graves, 1997).

Vários estudos confirmam a validade dessa teoria. Em trabalho realizado com 469 empresas listadas pela Standard and Poor's (indice S\&P500), Waddock e Graves (1997) revelam que o desempenho social depende de um desempenho financeiro anterior positivo. Preston e O’Bannon (1997), em estudo realizado com dados de 67 grandes empresas norte americanas, indicaram que o desempenho financeiro precede ou é contemporâneo do desempenho social; o estudo de Melo (2012), com 624 empresas listadas pela Stand and Poor's (índice S\&P 1000), identificou que o desempenho financeiro anterior impacta positivamente o desempenho social e que este impacto ocorre em diferentes intensidades em cada uma das dimensões do desempenho social.

Para testar a validade dos pressupostos dessa teoria, sugere-se analisar os níveis de desempenho financeiro de PMEs e aplicar a classificação do desempenho social em obrigatório ou espontâneo, de forma a permitir a comparação das empresas por desemenho financeiro semelhante (superior/inferior). Sob esse aspecto, esste trabalho formula a primeira proposição de pesquisa:

Proposição 1: Pequenas e médias empresas com desempenho financeiro anterior superior apresentam desempenho social espontâneo posterior maior do que PMEs com desempenho financeiro inferior.

Como afirma Melo (2012), falta à literatura evidenciar a magnitude das intensidades provocadas por um melhor desempenho financeiro em cada dimensão do desempenho social, bem como explicar quais as subcategorias de cada dimensão que são mais afetadas positivamente por um desempenho financeiro superior. Assim, visando fechar tal lacuna, pode-se derivar da primeira proposição uma proposição auxiliar, conforme descrita abaixo:

Proposição 1a: Desempenho financeiro anterior superior afeta em diferentes intensidades as subcategorias das dimensões do desempenho social de PMEs em período posterior.

Para Waddock e Graves (1997), enquanto empresas com desempenho financeiro superior direcionam investimentos para ações de RSE com impactos de longo prazo, como melhoria de escolas, empresas com problemas financeiros têm pequena capacidade para investir nesse tipo de atividade. Então, empresas com desempenho financeiro inferior não investem ou têm dificuldades para investir em práticas RSE espontâneas. E considerando os problemas financeiros das PMEs brasileiras, é razoável postular que PMEs nessa situação, limitam-se apenas a cumprir a lesgislação nas dimensões do desempenho social, ou seja, empreendem somente aquelas ações abarcadas pelo domínio legal da RSE e não apresentam desempenho social espontâneo, conforme descrito na proposição 2:

Proposição 2: Pequenas e médias empresas com desempenho financeiro anterior inferior apresentam apenas desempenho social obrigatório em período posterior.

\subsection{Implicações da Teoria Good Management}

Implícito no argumento de que fazer o "bom" (desempenho social) leva a fazer "bem" (desempenho 
financeiro) está a noção de que uma gestão eficaz das responsabilidades sociais e das relações com os stakeholders melhora a lucratividade da firma (Waddock \& Graves, 1997). A teoria Good Management parte dessa premissa e integra a base teórica de muitos estudos em responsabilidade social. Tal corrente teórica defende que se ações da RSE forem incorporadas à estratégia da empresa resultam em recompensas por parte da sociedade (Mcwilliams \& Siegel, 2000), tais como: melhor reputação da empresa perante seus consumidores (Brammer \& Pavelim, 2006), maior preferência e lealdade dos clientes para com seus produtos e serviços (McGuire, Sundgren, \& Schneeweis, 1988; Pivato, Misani, \& Tencati, 2008), maior motivação, satisfação e produtividade dos funcionários e menos problemas trabalhistas (Mcguire, et al., 1988; Waddock \& Graves, 1997).

Nesse aspecto, os pressupostos da teoria good management podem ser utilizados para explicar alguns dos resultados encontrados por estudos que investigam as relações entre desempenho financeiro e desempenho social empresarial. Van der Lann et al., (2008), Cesar e Silva-Júnior (2008) e Orellano e Quiota (2011) encontraram relação positiva entre desempenho financeiro e indicadores sociais internos (dimensão empregados) e negativa em relação aos indicadores sociais externos (dimensão comunidade). Esses resultados distintos podem ser explicados com base na importância do stakeholder para as empresas. Como já citado, empregados são stakeholders primários. Sua interdependência com a empresa é elevada devido às frequentes transações e relações de trocas, diretas e recíprocas, que eles mantêm com a organização (Van Der Lann et al., 2008).

Por outro lado, os resultados em relação à dimensão comunidade podem ser negativos porque as ações de RSE direcionadas a esse stakeholder, principalmente nas empresas brasileiras demonstram, em sua maior parte, ser assistencialistas, aleatórias e pulverizadas (Passador, 2002). Isso indica que a execução dessas ações pode ocorrer de forma indiscriminada, conforme apontado por Singal (2014). Entretanto, nem todas as ações nessa dimensão são executadas indiscriminadamente. Podem existir ações que justificam ser integradas à estratégia, como é o caso de práticas obrigatórias, relacionadas ao gerenciamento do impacto da empresa na comunidade, desenvolvidas em termos de tratamento de geração de lixo, mau cheiro, efluentes e outras formas de poluição.

Diante do exposto, conforme evidenciado por Waddock \& Graves (1997) e Singal (2014), a forma com que ações de RSE são executadas pelas empresas impacta positivamente ou negativamente o desempenho financeiro. Se forem integradas à estratégia podem melhorá-lo, mas se forem indiscriminadas podem piorá-lo. Com base nesses argumentos, pode-se afirmar que as categorias do desempenho social (identificadas na proposição 1a) afetadas mais intensamente de forma positiva por um desempenho financeiro superior, retornarão em melhor despenho financeiro posterior se forem estratégicas para o negócio. Se não o forem, destruirão o desempenho financeiro no período seguinte. Sob esse aspecto, são formuladas as proposições 3 e 4 :

Proposição 3: Categorias do desempenho social afetadas positivamente por desempenho fianceiro anterior superior impactam positivamente o desempenho financeiro posterior se forem estratégicas para as empresas.

Proposição 4: Categorias do desempenho social afetadas positivamente por desempenho fianceiro anterior superior impactam negativamente $\mathrm{o}$ desempenho financeiro posterior se não forem estratégicas (indiscriminadas) para as empresas.

\subsection{Modelo Conceitual}

A figura 2 esquematiza os contextos e as proposições de pesquisa. Para se medir as relações de causa e efeito entre desempenho financeiro e desempenho social é necessário um hiato de tempo, de $\mathrm{T}-1$ (período anterior) para $\mathrm{T}+1$ (período posterior). Assim, a proposta desse texto é que futuros estudos empíricos realizem pesquisas longitudinais, de forma a perceber melhor os impactos existentes. 
Desempenho Social e Financeiro de Pequenas e Médias Empresas: Modelo Conceitual de Causa e Efeito

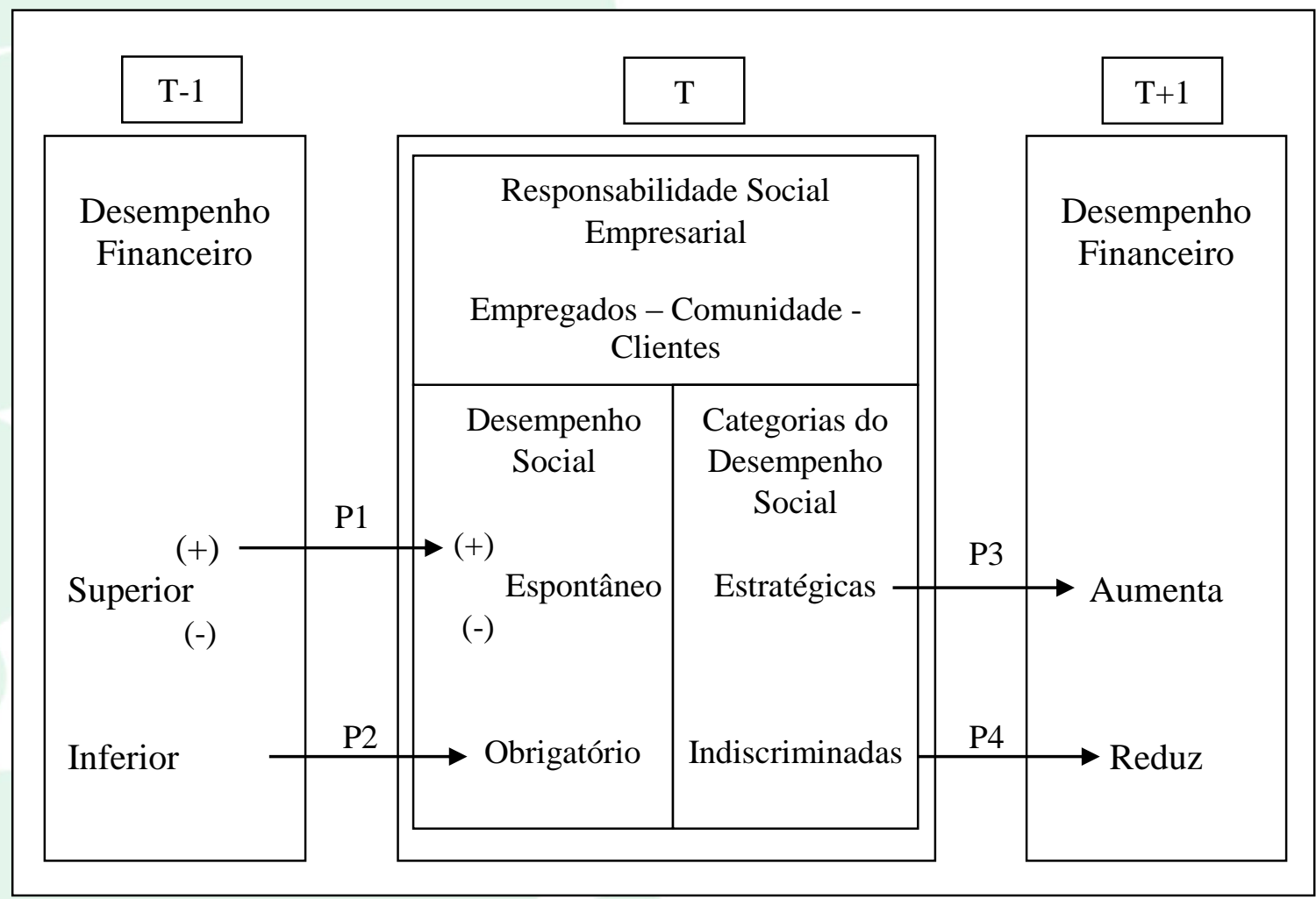

Figura 2: Modelo conceitual da pesquisa Fonte: o autor

\section{DISCUSSÕES E NOTAS FINAIS}

Visando contribuir com o avanço da literatura que tem examinado as relações entre desempenho social e financeiro empresarial, este texto teve por objetivo apresentar um modelo conceitual composto por proposições de pesquisas para serem testados por futuros trabalhos empíricos que desejem explorar o fenômeno em pequenas e médias empresas brasileiras. Baseado em trabalhos anteriores, foi proposto que o desempenho social seja mensurado nas dimensões mais próximas e relevantes de PMEs, que são empregados, clientes e comunidade. Isso permite avaliar até que ponto PMEs conseguem responder às demandas desses stakeholders.

Considerando as diferenças entre PMEs e empresas de grande porte e as limitações do modelo conceitual de RSE, de Schwartz e Carroll (2003), este texto sugeriu alterações na forma de classificação das ações de RSE para adequar o modelo ao contexto de PMEs. Ao classificar o desempenho social em obrigatório ou espontâneo, futuras pesquisas podem operacionalizar melhor o desempenho social e testar mais em profundidade a teoria slack-resources. Nesse aspecto, foi sugerido que as dificuldades financeiras de PMEs brasileiras também sejam levadas em conta para se mensurar os níveis de desempenho financeiro.
O teste da teoria slack-resources poderá ser realizado por meio das proposições 1, 1a e 2. Pela proposição 1, espera-se que PMEs com melhor desempenho fianceiro apresentem desempenho social além do obrigatório. Os resultados podem revelar se o pensamento de Milton Friedman, considerado fora de moda, ainda subsiste, conforme sugere Melo (2012). Ao testar a proposição 1a, espera-se que futuros estudos possam revelar que categorias ou temas das dimensões representativas dos stakeholders mais relevantes às PMEs são priorizadas pelas empresas com desempenho financeiro superior quanto à alocação de recursos, conforme sugestão de Melo (2012).

Testando a proposição 2 , futuros estudos podem identificar se apesar de dificuldades financeiras, PMEs apresentam, em algumas das dimensões, desempenho social espontâneo. Se isso for evidenciado, pode-se refutar parte dos pressupostos da teoria slack resources, uma vez que ela sozinha não seria suficiente para explicar maiores níveis de desempenho social em empresas com desempenho financeiro inferior. Ficaria demonstrado que o desempenho social também é influenciado por outros fatores vinculados à consciência ética e moral dos proprietários, conforme sugere Schwartz e Carroll (2003).

Os resultados dos testes das proposições 3 e 4 revelarão se o impacto sobre o desempenho financeiro, 
das categorias do desempenho social mais afetadas positivamente por desempenho financeiro superior, é qualificado pela forma de gestão. Se isso for evidenciado, os pressupostos da teoria good management serão reforçados. A implicação gerencial desse resultado é a necessidade de as empresas alinhar alocação de recursos e forma de gestão, caso estejam interessadas em auferir retornos financeiros a partir de um melhor desempenho social.

Dentre as limitações desse trabalho, destacase a articulação teórica, que foi realizada com apenas duas teorias da RSE - slack-resources e good management. Trabalhos empíricos ao testar as

\section{REFERÊNCIAS}

Barbieri, J. C., \& Cajazeira, J. E. R. (2009). Responsabilidade social empresarial e empresa sustentável: da teoria à prática. São Paulo: Saraiva.

Brammer, S. J., \& Pavelin, S. (2006). Corporate reputation and social performance: The importance of fit. Journal of Management Studies, 43(3), 435455.

Brammer, S., Hoejmose, S., \& Marchant, K. (2012). Environmental management in SMEs in the UK: practices, pressures and perceived benefits. Business Strategy and the Environment, 21(7), 423434.

Brigham, E. F., Houston, J. F. (1999). Fundamentos da Moderna Administração Financeira. Rio de Janeiro: Campus.

Buckley, P. J. (1989). Foreign direct investment by small and medium sized enterprises: The theoretical background. Small Business Economics, 1(2), 89100.

Campos, A. (2014). Metade das empresas brasileiras estão com dívidas em atraso. Economia \& Negócios. Jornal Estadão. 15.07.2014. Capturado de

http://economia.estadao.com.br/noticias/geral,meta de-das-empresas-brasileiras-esta-com-dividas-ematraso, 1560485 .

Campos, J. G. F., Nishimura, A. T., Ramos, H. R., Cherez, R. L., \& scalfi, V. B. (2008). As pequenas e médias empresas no Brasil e na China: uma análise comparativa. In: XI SEMEAD Empreendedorismo em Organizações. São Paulo. Recuperado de: http://www.ead.fea.usp.br/semead/11 semead/result ado/trabalhospdf/895.pdf. proposições aqui sugeridas podem expandir a articulação teórica incorporando discussões relativas à teoria dos stockholders (acionistas) e teoria dos stakeholders. Isso enriquecerá do ponto de vista conceitual, além de permitir discussões teóricas mais aprofundadas dos resultados. Futuros trabalhos também podem incluir na mensuração do desempenho social outras dimensões, como meio ambiente e governo. Esse aspecto fortalece a mensuração do mensuração do desempenho social e alinha-se ao que é realizado pela literatura internacional, que usa mensurações em cinco ou mais dimensões.

Carroll, A. B. (1979). A three-dimensional conceptual model of corporate performance. Academy of management review, 4(4), 497-505.

Carroll, A. B. (1991). The pyramid of corporate social responsibility: toward the moral management of organizational stakeholders. Business Horizons, 34(4), 39-48.

Cesar, J. F., \& Silva, J. A. (2008). A relação entre a responsabilidade social e ambiental com o desempenho financeiro: um estudo empírico na Bovespa no período de 1999 a 2006. In Congresso Associação Nacional dos Programas de PósGraduação em Ciências Contábeis (APCONT), 2, Anais. Salvador.

Combs, J. G.; Crook, T. R., \& Shook, C. L. (2005). The dimension of organizational performance and its implications for strategic management research. In Ketchen, D. J., \& Bergh, D. D. (Org). Research Methodology in Strategy and Management, 2, 259286. San Diego: Elsevier.

Crisóstomo, V. L., Freire, F. de S. \& Vasconcellos, F. C. de (2011). Corporate social responsibility, firm value and financial performance in Brazil. Social Responsibility Journal, 7(2), 295-309.

Esteves, M. L., Silva, S. S. da., \& Aligreri, L. M. (2007). Gestão estratégica da responsabilidade social: o caso das empresas do setor de atacado e comércio exterior na região sul do país. FAE, 10(1), 19-26.

Faria, A., \& Sauerbronn, F. F. (2008). A responsabilidade social é uma questão de estratégia? Uma abordagem crítica. Revista de Administração Pública, 42(1), 7-34.

Frederick, W. C. (1994). From CSR1 to CSR2 The maturing of business-and-society thought. Business \& Society, 33(2), 150-164. 
Griffin, J. J., \& Mahon, J. F. (1997). The corporate social performance and corporate financial performance debate twenty-five years of incomparable research. Business \& Society, 36(1), 5-31.

Jenkins, H. (2004). A critique of conventional CSR theory: An SME perspective. Journal of General Management, 29, 37-57.

Jenkins, H. (2006). Small business champions for corporate social responsibility. Journal of Business Ethics, 67(3), 241-256.

Koteski, M. A. (2004). As micro e pequenas empresas no contexto econômico brasileiro. Revista FAE Business, 8(1), 16-18.

Lucato, W. C., \& Vieira-Junior, M. (2006). As dificuldades de capitalização das pequenas e médias empresas brasileiras. Revista Produção, 16(1), 2433.

McGuire, J. B., Sundgren, A., \& Schneeweis, T. (1988). Corporate social responsibility and firm financial performance. Academy of management Journal, 31(4), 854-872.

Mcwilliams, A., \& Siegel, D. (2000). Corporate Social Responsibility And Financial Performance: Correlation Or Misspecification? Strategic Management Journal (21), 603-609.

Melo, T. (2012). Slack-resources hypothesis: a critical analysis under a multidimensional approach to corporate social performance. Social Responsibility Journal, 8(2), 257-269.

Mintzberg, H. (1973). Strategy making in three modes. Californian Management Review, 16(2).

Mintzberg, H., Lampel, J., Quinn, J. B., \& Ghoshal, S. (2006). O processo da estratégia: conceitos, contextos e casos selecionados, $4^{\mathrm{a}}$ ed. Porto Alegre: Brookman.

Oliveira, J. A. (1984). Responsabilidade social em pequenas e médias empresas. RAE, 24(4), 203-210.

Orellano, V. I. F., \& Quiota, S. (2011). Análise do retorno dos investimentos socioambientais das empresas brasileiras. RAE, 51(5), 471-484.

Passador, C. S. (2002). A responsabilidade social no Brasil: uma questão em andamento. In VII Congreso Internacional del CLAD sobre la Reforma del Estado y de la Administración Pública.
Lisboa. Recuperado de:

http://unpan1.un.org/intradoc/groups/public/docum ents/clad/clad0044201.pdf.

Pivato, S., Misani, N., \& Tencati, A. (2008). The impact of corporate social responsibility on consumer trust: the case of organic food. Business Ethics: A European Review, 17(1), 3-12.

Porter, M. E., \& Kramer, M. R. (2006). The link between competitive advantage and corporate social responsibility. Harvard business review, 84(12), 78-92.

Preston, L. E., \& O’bannon, D. P. (1997). The corporate social-financial performance relationship. Business and society, 36(4), 419-429.

Princic, L., \& Floyd, M. (2003). Engaging small business in corporate social responsibility: A Canadian small business perspective on CSR. Canadian Business for Social Responsibility, 21, 34-41.

Ramos-Rodríguez, A. R., \& Ruíz-Navarro, J. (2004). Changes in the intellectual structure of strategic management research: A bibliometric study of the Strategic Management Journal, 1980-2000. Strategic Management Journal, 25(10), 981-1004.

Raynard, P., \& Forstater, M. (2002). Corporate social responsibility: Implications for small and medium enterprises in developing countries. United Nations Industrial Development Organization (UNIDO). Vienna, 2002.

Rowley, T., \& Berman, S. (2000). A brand new brand of corporate social performance. Business \& society, 39(4), 397-418.

Ruf, B. M., Muralidhar, K., \& Paul, K. (1998). The development of a systematic, aggregate measure of corporate social performance. Journal of Management, 24(1), 119-133.

Schwartz, M. S., \& Carroll, A. B. (2003). Corporate social responsibility: A three-domain approach. Business Ethics Quarterly, 13(04), 503-530.

Serra, F. A. R., Albernaz, A., \& Ferreira, M. P. (2007). A Responsabilidade Social como Fator na Estratégia Internacional: O Estudo do Caso Natura. Revista Eletrônica de Administração, 13(4), 17-39.

Sethi, S. P. (1979). A conceptual framework for environmental analysis of social issues and evaluation of business response patterns. Academy of Management Review, 4(1), 63-74. 
Singal, M. (2014). Corporate social responsibility in the hospitality and tourism industry: Do family control and financial condition matter?. International Journal of Hospitality Management, 36, 81-89.

Spence, L. J. (1999). Does size matter? The state of the art in small business ethics. Business ethics: a European review, 8(3), 163-174.

Van der Laan, G., Van Ees, H., \& Van Witteloostuijn, A. (2008). Corporate social and financial performance: An extended stakeholder theory, and empirical test with accounting measures. Journal of Business Ethics, 79(3), 299-310.

Vo, L. C. (2011). Corporate social responsibility and SMEs: a literature review and agenda for future research. Problems and Perspectives in Management, 9(4), 89-97.
Volpon, C. T., \& Macedo-Soares, T. D. L. V. (2007). Alinhamento estratégico da responsabilidade socioambiental corporativa em empresas que atuam em redes de relacionamento: resultados de pesquisa na Petrobras. Revista de Administração Pública, 41(3), 391-418.

Waddock, S. A., \& Graves, S. B. (1997). The corporate social performance-financial performance link. Strategic management journal, 18(4), 303-319.

Wartick, S. L., \& Cochran, P. L. (1985). The evolution of the corporate social performance model. Academy of management review, 10(4), 758-769.

Wood, D. J. (1991). Corporate social performance revisited. Academy of management review, 16(4), $691-718$ 